\title{
UX-QUALI-MAP: uma ferramenta de mapeamento da atividade focada no suporte ao design para experiência
}

\section{UX-QUALI-MAP: an activity-mapping tool focused on supporting design for experience}

\author{
Genilda Oliveira de Araujo, Universidade Federal de Santa Catarina. \\ genilda@gmail.com
}

Lizandra Garcia Lupi Vergara, Universidade Federal de Santa Catarina. 1.vergara@ufsc.br

\section{Resumo}

O conceito de experiência do usuário é essencial para o design centrado no ser humano. Em contrapartida, por envolver tanto aspectos pragmáticos quanto subjetivos, sua abordagem requer um conhecimento mais profundo sobre os usuários e sobre o uso do produto. Considerando isso, esta pesquisa propõe uma ferramenta de mapeamento da atividade que visa estimular uma visão sistêmica dos aspectos afetivos, racionais e sensório-motores que o produto deve atender considerando os indivíduos e os ambientes organizacional e sociocultural da atividade. A construção da ferramenta baseou-se no relacionamento conceitual entre a teoria da atividade e a teoria de affordances. A avaliação da ferramenta foi feita com um grupo de quarenta e cinco alunos de graduação em design. $\mathrm{O}$ resultado demonstrou o seu potencial didático de contribuir para a compreensão do domínio do problema.

Palavras-chave: design de interação, ergonomia, experiência do usuário, affordances, teoria da atividade

\begin{abstract}
The concept of user experience is essential for human-centered design. On the other hand, because it involves both pragmatic and subjective aspects, its approach requires a deeper knowledge about users and about the use of the product. Considering these issues, this research proposes an activity mapping tool that aims to stimulate a systemic view of the affective, rational and sensory-motor aspects that the product must meet considering the individuals and the organizational and socio-cultural environments of the activity. The tool construction was based on the conceptual relationship between activity theory and affordances theory. The tool evaluation was done with a group of forty-five undergraduate students of design. The result demonstrated its didactic potential to contribute to the understanding of the problem domain.
\end{abstract}

Keywords: interaction design, ergonomics, user experience, affordances, activity theory 


\section{Introdução}

O conceito de experiência do usuário é essencial para o design centrado no ser humano. Sua adoção representa a passagem de uma abordagem pragmática de projeto, focada na eficácia e na eficiência da execução de tarefas, para uma abordagem mais ampla, que também contempla os aspectos subjetivos do uso de um produto. Tais aspectos enfatizam o bem-estar psicológico das pessoas, relacionando-se à busca por desenvolvimento pessoal, expressão individual e evocação de memórias importantes (HASSENZAHL, 2004).

Em contrapartida, a abordagem desses aspectos subjetivos requer um conhecimento mais profundo sobre os usuários e sobre o uso do produto. De acordo com a norma ISO 9241-210 (2018), a experiência do usuário pode ser definida como o conjunto de "percepções e respostas de uma pessoa resultantes do uso elou do uso antecipado de um produto, sistema ou serviço". Além disso, inclui "todas as emoções, crenças, preferências, percepções, respostas físicas e psicológicas dos usuários, comportamentos e realizações que ocorrem antes, durante e após o uso". Assim, a experiência é um consequente da relação que se estabelece entre usuário e produto durante o uso. Para melhorar sua qualidade, é preciso ajustar essa relação.

Um caminho para esse ajuste é a abordagem da atividade. De acordo com Hassenzahl et al. (2013), experiências positivas estão ligadas à realização de atividades significativas para os usuários e o papel dos produtos é dar suporte a essas atividades. Desta forma, por meio da compreensão da atividade, é possível criar produtos que atendam melhor aos usuários e sejam mais propensos a contribuir para experiências positivas. Neste âmbito, outro conceito importante é o de affordances, que descreve os potenciais para ação que decorrem da relação entre um indivíduo e os objetos de um ambiente (GIBSON, 1979). Ou seja, affordances abordam a interdependência ou complementaridade entre ação humana, produtos e ambiente. Nesse sentido, de acordo com Galvao e Sato (2005), é essencial a inclusão de estudos dos usuários combinados com o pensamento de affordances para a geração da arquitetura funcional de um produto.

Com base no exposto acima, esta pesquisa propõe uma ferramenta de mapeamento da atividade que visa estimular uma visão sistêmica dos aspectos afetivos, racionais e sensóriomotores que o produto deve atender considerando os indivíduos e os ambientes organizacional e sociocultural da atividade. A construção da ferramenta baseou-se no relacionamento conceitual entre a teoria da atividade e a teoria de affordances. A avaliação da ferramenta foi feita com um grupo de quarenta e cinco alunos de graduação em design. $\mathrm{O}$ resultado demonstrou o seu potencial didático de contribuir para a compreensão do domínio do problema.

\section{Fundamentação teórica}

Esta seção apresenta aspectos essenciais da teoria da atividade e da teoria de affordances. 


\subsection{Teoria da Atividade}

A teoria da atividade tem como conceito básico a noção de que toda atividade humana se origina a partir de uma necessidade não satisfeita (LEONT'EV, 1978). Essa conexão entre atividade e necessidade é o que transforma a atividade no cenário a ser compreendido para que se possa projetar com foco na experiência do usuário. Segundo Hassenzahl et al. (2013), é o preenchimento ou a frustração de necessidades que torna uma experiência positiva ou negativa, além de conferir-lhe significado pessoal.

Este trabalho usa a estrutura da atividade (LEONT'EV, 1978) como ferramenta para auxiliar na compreensão da atividade humana. Esta estrutura, mostrada na figura 1, é um modelo hierárquico composto por três níveis de abstração que abordam os aspectos afetivo, racional e sensório-motor da atividade. Por relacionar esses níveis, permite visualizar a atividade como um sistema no qual motivação, cognição e comportamento são integrados e organizados por mecanismos de auto-regulação para o alcance de metas (BEDNY; KARWOWSKI, 2004).

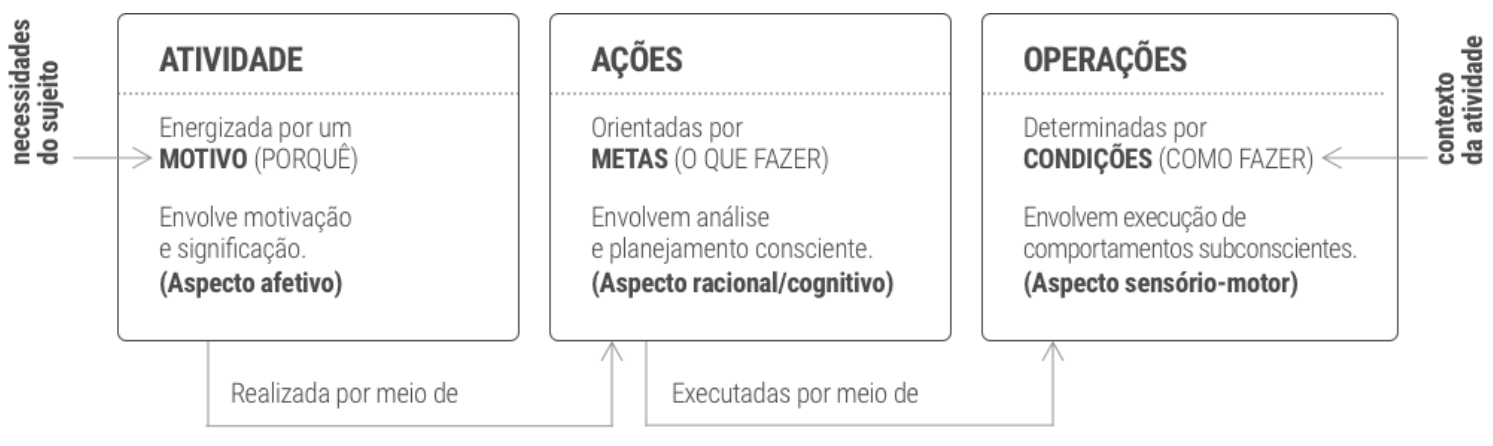

Figura 1 - Estrutura da Atividade. Fonte: elaborada pelas autoras.

O nível mais alto da abstração foca na atividade como um todo e aborda o seu propósito, que consiste na satisfação de alguma necessidade. É esta necessidade que dá razão de existência à atividade, atuando como seu motivo energizante (porquê). Segundo Leont'ev (1978), a função do motivo é ajudar o indivíduo a avaliar o significado das circunstâncias e dos resultados de suas ações nessas circunstâncias, dotando-os de um senso pessoal e de afeto. Assim, quanto mais significativa for a necessidade para o indivíduo e quanto maiores forem as expectativas de satisfação e os resultados efetivamente obtidos durante a atividade, mais positivos serão os afetos e maior o engajamento afetivo com a atividade.

No segundo nível, estão as ações, que são os processos planejados para satisfazer a necessidade. Nele, o indivíduo realiza uma análise e define uma meta geral do que será feito para satisfazer a necessidade (o que). Por exemplo, para satisfazer à necessidade de descanso físico e mental, a meta pode ser a de fazer uma viagem para uma região praiana. Com base nessa meta geral, são planejadas as ações. Por exemplo, no caso da viagem, as ações podem incluir compra de passagem aérea, aluguel de carro e reserva de hotel. Cada ação, por sua vez, pode ter uma meta específica que lhe orientará (LEONT'EV, 1978). 
Por fim, completando a estrutura da atividade, cada ação pode ser decomposta em operações, que estabelecem os passos concretos para sua execução (como). Estas operações não são planejadas pelo indivíduo, mas realizadas de acordo com as condições dadas pelo contexto, que inclui os produtos usados. Por exemplo, na compra de passagem aérea, a indicação das datas de partida e de retorno será feita de acordo com os componentes da interface do site ou do aplicativo. Ou seja, a interface estabelece as condições interativas, definindo, por exemplo, se o usuário deverá digitar as datas ou selecioná-las em uma calendário. Estas condições podem ser detectadas e tratadas de forma automática pelo sistema sensório-motor (LEONT'EV, 1978), com base na sua capacidade de aplicar de forma subconsciente rotinas e habilidades aprendidas. Por exemplo, um indivíduo com prática em digitação não precisa controlar conscientemente as operações de toque nas teclas, que são feitas de modo automático.

\subsection{Affordances}

Este trabalho adota o conceito de affordances para abordar a forma como um produto pode dar suporte à atividade do usuário, ou seja, as oportunidades que pode oferecer. Segundo Gibson (1979), autor da teoria de affordances, as oportunidades de ação nascem da complementaridade entre um ator e os objetos de um ambiente. Assim, é importante entender que affordances não são propriedades dos objetos. Emergem com base na relação entre as propriedades dos objetos em um certo ambiente e as características dos atores. Por exemplo, uma porta oferece o affordance de abrir a uma pessoa com estatura e habilidades físicas compatíveis, mas não a uma criança pequena.

Para Bærentsen e Trettvik (2002), embora o surgimento de affordances dependa das características dos produtos, estas características apenas originam affordances quando os sujeitos as relacionam à sua atividade, sendo, por isso, propriedades de sistemas de atividades. Nesse sentido, a lente dos affordances ajuda a abordar os produtos no contexto dinâmico da atividade. Permite analisar e descrever as diferentes oportunidades que um produto deve prover a certos usuários no contexto de uma certa atividade. Além disso, também permite abordar os aspectos que se complementam entre usuário, produto e atividade para que tais oportunidades estejam disponíveis.

\section{Procedimentos metodológicos}

Para desenvolver o relacionamento conceitual entre as teorias da atividade e de affordances, foram realizadas duas revisões sistemáticas de literatura. $\mathrm{O}$ foco dessas revisões foi identificar esquemas classificatórios de affordances e verificar como os tipos de affordances propostos relacionam-se aos níveis da estrutura da atividade. Foi utilizada a metodologia de Kitchenham e Charters (2007) que estabelece cinco passos: (a) definição da pergunta de pesquisa; (b) definição da estratégia de busca; (c) busca; (d) seleção dos resultados primários; (e) extração de dados. Ambas as revisões usaram as bases de dados SCOPUS, ACM Digital Library e Web of Science. 
A primeira revisão teve como pergunta de pesquisa: "Quais são os esquemas classificatórios de affordances baseados na teoria da atividade?". Foram incluídos na busca tanto artigos de revistas quanto de conferências. A tabela 1 apresenta um panorama quantitativo da revisão ${ }^{1}$.

\begin{tabular}{lccc} 
Data Base & Resultados & Não Repetidos & Selecionados \\
SCOPUS & 29 & 29 & 5 \\
ACM Digital & 5 & 2 & 1 \\
Web of Science & 38 & 26 & 0 \\
Total & $\mathbf{7 2}$ & $\mathbf{5 7}$ & $\mathbf{6}$ \\
\hline
\end{tabular}

Tabela 1. Panorama quantitativo da primeira revisão sistemática. Fonte: elaborada pelas autoras.

A segunda revisão teve como pergunta de pesquisa "Quais são os esquemas classificatórios de affordances não baseados na teoria da atividade?". O propósito foi verificar se estes esquemas também se alinham aos níveis da estrutura da atividade. A tabela 2 apresenta um panorama quantitativo da revisão ${ }^{2}$.

\begin{tabular}{lccc} 
Data Base & Resultados & Não Repetidos & Selecionados \\
\hline SCOPUS & 91 & 91 & 19 \\
ACM Digital & 8 & 8 & 0 \\
Web of Science & 43 & 14 & 1 \\
Total & $\mathbf{1 4 2}$ & $\mathbf{1 1 3}$ & $\mathbf{2 0}$ \\
\hline
\end{tabular}

Tabela 2. Panorama quantitativo da segunda revisão sistemática. Fonte: elaborada pelas autoras.

A extração de dados começou com um mapeamento entre os esquemas classificatórios encontrados e a estrutura da atividade. Neste processo, a descrição de cada categoria de cada esquema foi comparada à caracterização dos níveis da estrutura da atividade. Como resultado, cada categoria de affordance foi associada a um nível da estrutura da atividade. A partir desse mapeamento, foram extraídas os dados para montagem da ferramenta.

A avaliação da ferramenta foi feita com base na sua aplicação por alunos de graduação em design. O procedimento de avaliação foi submetido à apreciação ética, recebendo o certificado de aprovação CAAE n ${ }^{\circ}$ 01938018.9.0000.0121. O critério de inclusão de participantes foi que estivessem cursando as disciplinas de Ergonomia, Usabilidade ou Projeto de Interfaces. Os participantes aplicaram a ferramenta na geração de um roteiro de pesquisa para um projeto de aplicativo. Após a atividade, preencheram um questionário de avaliação da ferramenta. Por fim, os questionários foram recolhidos de forma anônima.

\footnotetext{
1 A string de busca da primeira revisão continha as palavras-chave ("activity theory") AND ("affordances"). Em função do pequeno número de resultados, optou-se por não incluir termos restritivos, nem limitar o período de publicação.

${ }^{2}$ A string de busca da segunda revisão foi: ("affordances") AND ("model" or "framework" or "hierarchy" or "levels" or "types" or "typology" or "classification") AND ("human computer interaction" or "user interfaces" or "product design"). Neste caso, o uso apenas do termo affordances gerou muitos resultados (cerca de seis mil) e demandou um refinamento da estratégia de busca. Assim, foram introduzidas as palavras-chave "model", "framework", "hierarchy", "levels", "types", "typology" e "classification". Além disso, optou-se por adicionar as palavras-chave "human computer interaction", "user interfaces" ou "product design". Por fim, manteve-se a opção de não limitar o período de publicação.
} 


\section{Resultados}

Esta seção apresenta os resultados obtidos, que incluem o relacionamento da estrutura da atividade e affordances, a montagem da ferramenta e o resultado do processo de avaliação.

\subsection{Relacionamento entre estrutura da atividade e affordances}

Para relacionar estrutura da atividade e esquemas classificatórios de affordances, cada tipo de affordance de cada esquema foi associado a um nível da estrutura da atividade, conforme mostrado na tabela 3. Durante esse processo, foram identificados três grupos de trabalhos, apontados pelas letras $\mathrm{A}, \mathrm{B}$ e $\mathrm{C}$ na última coluna da tabela 3. O grupo $\mathrm{A}$ inclui esquemas que cobrem os três níveis da atividade. O grupo $\mathrm{B}$ contém esquemas que se focam em ações e operações e não abordam a dimensão motivacional. $\mathrm{O}$ grupo $\mathrm{C}$ contém esquemas que se centram na descrição de apenas um nível da estrutura da atividade.

\begin{tabular}{|c|c|c|c|c|c|c|}
\hline Autores & Nível da Atividade & \multicolumn{2}{|c|}{ Nível das ações } & \multicolumn{2}{|c|}{ Nível das operações } & \\
\hline Vicente e Rasmussen (1990) & Porque & \multicolumn{2}{|l|}{$\mathrm{O}$ que } & \multicolumn{2}{|l|}{ Como } & A \\
\hline Bærentsen e Trettvik (2002) & $\begin{array}{l}\text { Ligados a } \\
\text { necessidades }\end{array}$ & \multicolumn{2}{|l|}{ Instrumentais } & \multicolumn{2}{|c|}{ Operacionais } & \\
\hline Turner e Turner (2002) & Culturais & \multicolumn{2}{|l|}{ Para tarefas } & \multicolumn{2}{|c|}{ De usabilidade } & \\
\hline $\begin{array}{l}\text { Sun (2007); } \\
\text { Sun e Hart-Davidson (2014) }\end{array}$ & Sociais & \multicolumn{2}{|l|}{ Instrumentais } & \multicolumn{2}{|c|}{ Operacionais } & \\
\hline Sharritt (2010) & Motivacionais & \multicolumn{2}{|c|}{ Para realização de metas } & \multicolumn{2}{|c|}{ Operações perceptíveis } & \\
\hline Pols (2012) & Para atividade & Para uso & Para efeito & \multicolumn{2}{|c|}{ Para manipulação } & \\
\hline Pucillo e Cascini (2014) & Para experiência & \multirow{2}{*}{\multicolumn{2}{|c|}{ Para efeito }} & \multicolumn{2}{|c|}{ Para manipulação } & \\
\hline Vyas et al. $(2017 ; 2006)$ & Em articulação & & & \multicolumn{2}{|c|}{ Em informação } & \\
\hline Zhao et al. (2013) & Afetivos & \multicolumn{2}{|c|}{ Cognitivos e controle } & \multicolumn{2}{|l|}{ Físicos } & \\
\hline \multirow[t]{2}{*}{ Rozycki et al. (2012) } & \multicolumn{3}{|l|}{ Psicológicos e Sociais } & \multirow{2}{*}{\multicolumn{2}{|c|}{ Corpo físico e ambiente físico }} & \\
\hline & Funcionais & & & & & \\
\hline Ciavola e Gershenson (2016) & & - & Para efeito & \multicolumn{2}{|c|}{ Para manipulação } & \multirow[t]{5}{*}{$\mathrm{B}$} \\
\hline Grange e Benbasat (2011) & - & Para ação & Funcionais & Estruturais & & \\
\hline Kaptelinin e Nardi (2012) & - & Instrumentais & Para efeito & Para manipu & lação & \\
\hline Jonietz e Timpf (2015) & - & Pragmáticos & Semânticos & Para realizac & & \\
\hline Hartson (2003) & - & \multicolumn{2}{|c|}{ Cognitivos e funcionais } & \multicolumn{2}{|c|}{ Físicos e Sensoriais } & \\
\hline Turner (2005) & $t$ & - & & Complexos & Simples & \multirow[t]{4}{*}{$\mathrm{C}$} \\
\hline Zhang (2008) & Motivacionais & - & & & - & \\
\hline Nahl (2007) & $\begin{aligned} \\
\end{aligned}$ & - & & \multicolumn{2}{|c|}{ Para recepção e uso } & \\
\hline Zhang e Patel (2008) & & & & Cognitivos & $\begin{array}{l}\text { Biológicos, } \\
\text { Físicos e } \\
\text { Perceptuais }\end{array}$ & \\
\hline
\end{tabular}

Tabela 3. Tipos de affordances relacionados à estrutura da atividade. Fonte: elaborada pelas autoras.

Com o propósito de ilustrar os esquemas classificatórios, são apresentados três autores. O esquema de Bærentsen e Trettvik (2002) propõe affordances operacionais, ligados à forma de execução de ações e ao uso de ferramentas; affordances instrumentais, que atuam como instrumento para a realização de ações e o alcance de metas; bem como affordances ligados à satisfação de necessidades. O esquema de Turner e Turner (2002) descreve affordances para usabilidade, ligados à interação com os controles das interfaces; affordances para tarefas, ligados ao alcance de metas dos usuários; bem como affordances culturais ligados aos aspectos 
culturais e motivacionais da atividade. Por fim, Kaptelinin e Nardi (2012) abordam affordances para manipulação, que permitem interagir com a tecnologia (p. ex.: pressionar uma tecla); affordances para efeitos, que descrevem o resultado intencional da manipulação (p. ex.: digitar uma letra); affordances para uso, que reúnem os efeitos e permitem agir por meio da tecnologia (p. ex.: escrever um texto).

Para consolidação teórica do relacionamento entre estrutura da atividade e affordances, a tabela 3 foi percorrida por coluna, ou seja, por nível da estrutura da atividade. Neste processo, buscou-se identificar com base nos diferentes tipos de affordances: (a) quais oportunidades podem ser oferecidas por um produto naquele nível; (b) quais elementos se relacionam para que essas oportunidades existam, ou seja, qual a complementaridade requerida naquele nível. Os itens oportunidade e complementaridade fazem parte do conceito original de affordances. Desta forma, esta consolidação destaca a forma como os elementos conceituais básicos da teoria de affordances podem ser descritos em cada nível da estrutura da atividade. O resultado da consolidação é apresentado a seguir.

\subsubsection{Tipos de affordances para atividade}

Esta seção traz uma descrição das três categorias de oportunidades que um produto deve oferecer para potencializar a atividade e a experiência humana:

- Oportunidades de experiência: representam o potencial que o uso do produto tem de satisfazer as necessidades que motivam a atividade. Estas oportunidades estão ligadas à realização pessoal dos usuários e à atribuição de significado à atividade (BÆRENTSEN; TRETTVIK, 2002). Sua percepção determina a força motivacional para ação (NAHL, 2007) e a gratificação do usuário (SHARRITT, 2010). Também estimulam reações afetivas por parte dos usuários (ZHAO et al., 2013) e dotam o produto de valores que expressam sua adequação ao propósito da atividade (TURNER; TURNER, 2002).

- Oportunidades de realização de tarefas: representam o potencial que as funcionalidades do produto têm de permitir a realização de tarefas e o alcance de metas. Assim, ligam o produto ao domínio das tarefas e permitem realizar algo neste contexto (GRANGE; BENBASAT, 2011). Seu foco é a instrumentalização da ação, auxiliando a estruturar e a melhorar o processo de ação dos usuários (BÆRENTSEN; TRETTVIK, 2002). Tem um caráter pragmático e fazem parte da dimensão racional da atividade humana.

- Oportunidades de manipulação: representam o potencial que a interface do produto tem de ser manipulada considerando as condições de uso. Estão ligadas à forma concreta de execução da ação (BÆRENTSEN; TRETTVIK, 2002). Assim, estes affordances permitem ao usuário executar algo em termos físicos (HARTSON, 2003). Por exemplo, incluem oportunidades do usuário tocar, arrastar e soltar elementos da interface. Estas oportunidades estão ligadas à dimensão sensório-motora da atividade. Seu controle pode ser feito de forma automática (BÆRENTSEN; TRETTVIK, 2002), com base na capacidade do sistema sensório-motor de aplicar de forma subconsciente rotinas e 
habilidades aprendidas. Desta forma, o usuário é liberado de ter que tratar analiticamente a manipulação física do produto.

Essas três categorias de oportunidades, representadas na figura 2, formam uma hierarquia do tipo meios-fins (VICENTE; RASMUSSEN, 1990), na qual os níveis inferiores são meios para realizar os níveis superiores. Desta forma, as oportunidades de manipulação permitem aproveitar as oportunidades de realizar de tarefas que, por sua vez, permitem aproveitar as oportunidades de experiência.

\begin{tabular}{|l|l|l|l|}
\hline $\begin{array}{l}\text { AFFORDANCES } \\
\text { 繇 }\end{array}$ & $\begin{array}{l}\text { Oportunidades } \\
\text { de experiência } \\
\text { Engajamento afetivo. }\end{array}$ & $\begin{array}{l}\text { Oportunidades de } \\
\text { realização de tarefas } \\
\text { Engajamento racional. }\end{array}$ & $\begin{array}{l}\text { Oportunidades } \\
\text { de manipulação } \\
\text { Engajamento sensório-motor }\end{array}$ \\
\hline & meio pra & \multicolumn{2}{|c|}{ meio pra } \\
\hline
\end{tabular}

Figura 2 - Hierarquia de affordances. Fonte: elaborada pelas autoras.

\subsubsection{Complementaridade para affordances}

Ao falar de complementaridade, Gibson aborda a relação entre ator, ambiente físico e produtos. O trabalho de Rozycki et al. (2012) expande essa visão e descreve que o ambiente deve ser considerado tanto em sua dimensão física, com suas características materiais, quanto em sua dimensão abstrata, que envolve as características socioculturais. Além disso, também propõe que o elemento humano deve ser considerado tanto na dimensão individual quanto na dimensão de grupo. Essa abordagem do coletivo é ampliada no trabalho de Vyas et al. (2017), que propõe três níveis: o indivíduo, o organizacional e o sociocultural. Neste trabalho, estas dimensões de complementaridade foram combinadas com a hierarquia de affordances, conforme mostrado na figura 3 .

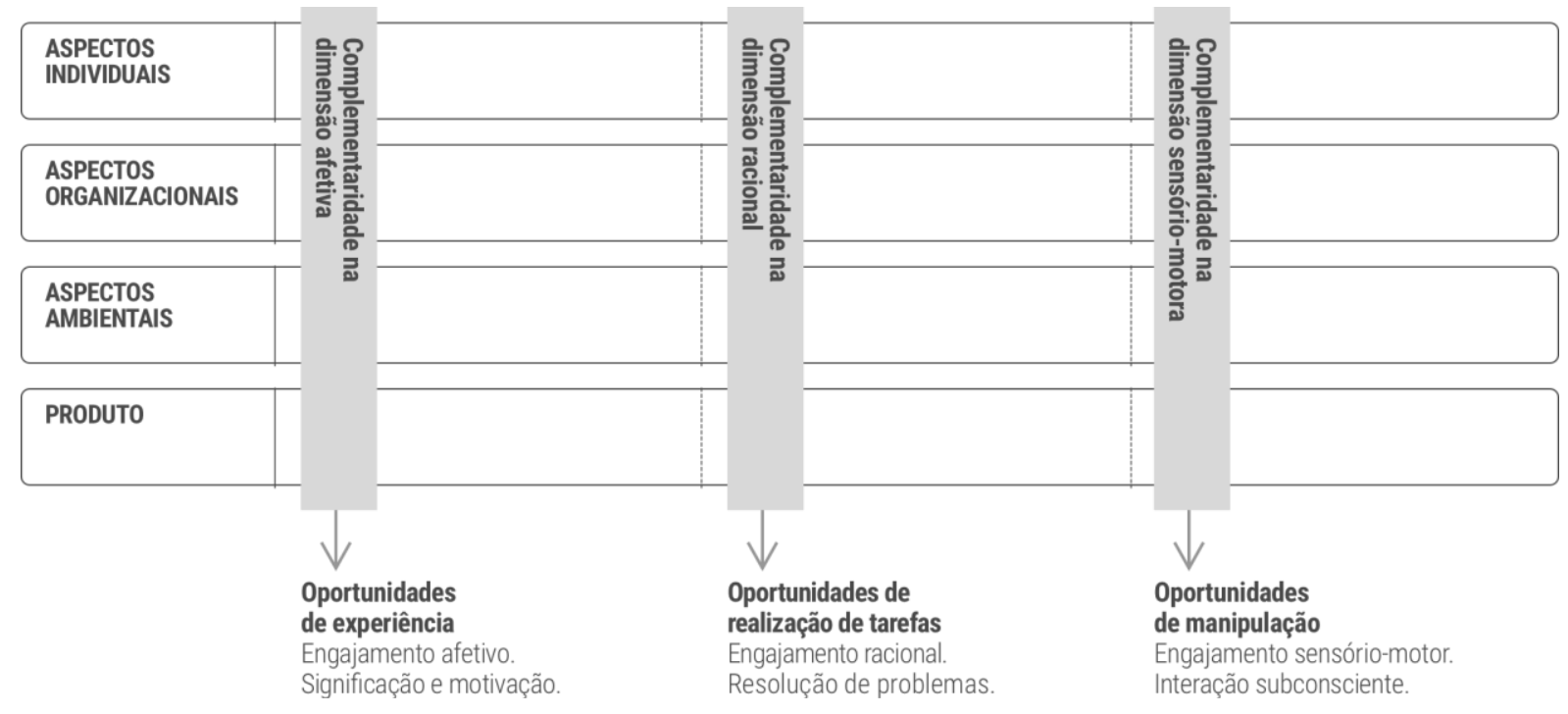

Figura 3 - Complementaridade para affordances. Fonte: elaborada pelas autoras. 
Nos affordances para experiência, o ponto central da complementaridade diz respeito à relação entre os benefícios do uso do produto e a satisfação de necessidades (VYAS; CHISALITA; VAN DER VEER, 2006). Esse alinhamento pode ser necessário em vários níveis, pois a ação de um indivíduo pode estar direcionada à satisfação de suas necessidades individuais, de terceiros, de organizações ou sociais. Desta forma, é preciso compreender essa hierarquia de necessidades para identificar os benefícios que o uso do produto deve oferecer. Por exemplo, em um aplicativo de taxi como o 99, é preciso alinhar as necessidades dos motoristas (p. ex.: uma boa renda), dos usuários (p. ex.: transporte eficiente, seguro e com boa relação custo-benefício), da cooperativa de táxi (p. ex.: lucratividade) e sociais (p. ex.: melhoria da mobilidade urbana). Adicionalmente, o produto também deve apresentar complementaridade em relação a fatores socioculturais. Segundo Vyas et al. (2017) é importante observar a influência de princípios e convicções dos usuários, da cultura organizacional (que inclui valores e missão institucionais), das relações de poder e de hierarquia nas organizações, de normas sociais e culturais, bem como de condições interpretativas que incluem significados já atribuídos, preferências e atitudes em relação à tecnologia e à atividade.

Nos affordances para realização de tarefas, o ponto central da complementaridade diz respeito à relação entre as funcionalidades e o suporte ao plano de ação do usuário (POLS, 2012). Ciavola e Gershenson (2016) descrevem o plano de ação com uma hierarquia de metas, formada por uma meta central e um conjunto de sub-metas paralelas ou sequenciais. Além disso, as metas individuais podem estar alinhadas e dar suporte a metas organizacionais e também a metas sociais. Desta forma, deve-se compreender a hierarquia completa para definir as funcionalidades. Retomando o exemplo do aplicativo de táxi 99, é preciso alinhar as metas dos motoristas (p. ex.: aceitar o máximo de corridas próximas), dos usuários (p. ex.: encontrar rapidamente um bom motorista por um preço justo), dos da cooperativa de táxi (p. ex.: conquistar e fidelizar usuários) e sociais (p. ex.: reduzir o número de carros em circulação estimulando o transporte compartilhado). Em sistemas voltados para o ambiente corporativo, é preciso manter a complementaridade da distribuição das funcionalidades entre os tipos de usuário com a organização de papéis dentro da organização e a distribuição horizontal de tarefas (VYAS; CHISALITA; DIX, 2017), bem como com o fluxo de trabalho colaborativo. Por fim, as funcionalidades também devem ser compatíveis com a estrutura de ação esperada. Para isso, devem ser observadas as práticas adotadas em nível individual, organizacional e social (ROZYCKI; KELLER; CYBULSKI, 2012). Ou seja, devem ser identificados procedimentos individuais, procedimentos institucionalizados, práticas de mercado, convenções sociais, bem como leis e regulamentações que estejam relacionadas à atividade. Por fim, Vyas et al. (2017) destaca que o nível de conhecimento do usuário sobre a tarefa também é um fator que deve ser considerado na formulação das funcionalidades.

Nos affordances para manipulação, o ponto central da complementaridade diz respeito à relação física entre indivíduo, produto, organização e ambiente. Essa relação faz uso dos potenciais do sistema sensório-motor, que pode controlar de modo subconsciente os movimentos do usuário, bem como pode disparar certos padrões de comportamento apropriados (BÆRENTSEN; TRETTVIK, 2002). Tais padrões abordam às formas de uso das interfaces e correspondem a convenções interativas aprendidas (ZHANG; PATEL, 2008). Por exemplo, com a repetição, os usuários interiorizam que a ação física associada a um botão é pressionar. Desta 
forma, quando encontram um botão, não precisam refletir sobre a sua manipulação. Considerando essas questões, a manipulação proposta pela interface do produto deve ser compatível com o nível de habilidade do usuário e com o seu repertório de padrões interativos. Além disso, o produto deve ser morfologicamente adequado aos usuários. Adicionalmente, a complementaridade manipulativa deve considerar as influências das condições físicas do local da atividade, tais como layout, luminosidade, temperatura e nível das ruído, além de condicionantes interativos como duração da atividade, ritmo da manipulação, desgaste do usuário, etc. Por exemplo, na versão do aplicativo 99 para o motorista, deve ser considerado que o mesmo será manipulado enquanto o usuário dirige, o que requer uma interface simplificada. Assim, a complementaridade neste nível envolve o tratamento de questões ergonômicas e de usabilidade em nível físico (TURNER; TURNER, 2002). Por fim, no caso de sistemas digitais, também devem ser consideradas condicionantes de natureza tecnológica, como, por exemplo, dispositivos computacionais que os usuários dispõe para a tarefa, outros sistemas com os quais o produto precisa se comunicar, infraestrutura de rede/wi-fi da organização ou do ambiente.

\subsection{Ferramenta proposta}

Os tipos de affordances para atividade e os seus requisitos de complementaridade foram sintetizados na ferramenta mostrada na figura 4 . O propósito dessa ferramenta é estimular uma visão sistêmica dos aspectos afetivos, racionais e sensório-motores que o produto deve atender considerando os níveis individual, organizacional e sociocultural da atividade. A forma de utilização sugerida envolve três etapas, indicadas pelas letras A, B e C.

As duas primeiras etapas direcionam a organização do conhecimento sobre o domínio do problema, incluindo a identificação das informações que o designer dispõe no início do levantamento de dados, a identificação das informações desconhecidos, bem como a geração de hipóteses para esses itens. A etapa A foca em estimular a visualização geral da estrutura da atividade. Nela, o designer deve identificar todos os envolvidos na atividade, incluindo indivíduos em diferentes papéis (p. ex.: no Uber, passageiro e motorista), bem como organizações e ambientes onde os indivíduos de cada papel estão inseridos. A seguir, para cada usuário, organização e ambiente devem ser identificadas a necessidade, a meta geral e as condições interativas. Com base nesses elementos, é possível começar a visualizar o escopo de aplicação do produto. A etapa B foca no aprofundamento dessa visão inicial por meio do mapeamento das características dos usuários e do contexto. Estimula a abordagem dos aspectos individuais, organizacionais e ambientais que o produto deve contemplar, conforme discutido na seção anterior do artigo.

A etapa $\mathrm{C}$ consiste na elaboração do roteiro de pesquisa. Para isso, devem ser revisados todos os aspectos identificados e/ou deixados em branco para cada papel, tipo de organização e ambiente. A partir deles, o designer pode elaborar questões sobre o que se deseja pesquisar (itens desconhecidos) ou validar (hipóteses geradas). 


\begin{tabular}{l|l|l} 
MAPEAMENTO & $\bigcirc$ DIMENSÃO & $\begin{array}{l}\text { DIMENSÃO } \\
\text { DAFETIVA }\end{array}$
\end{tabular}

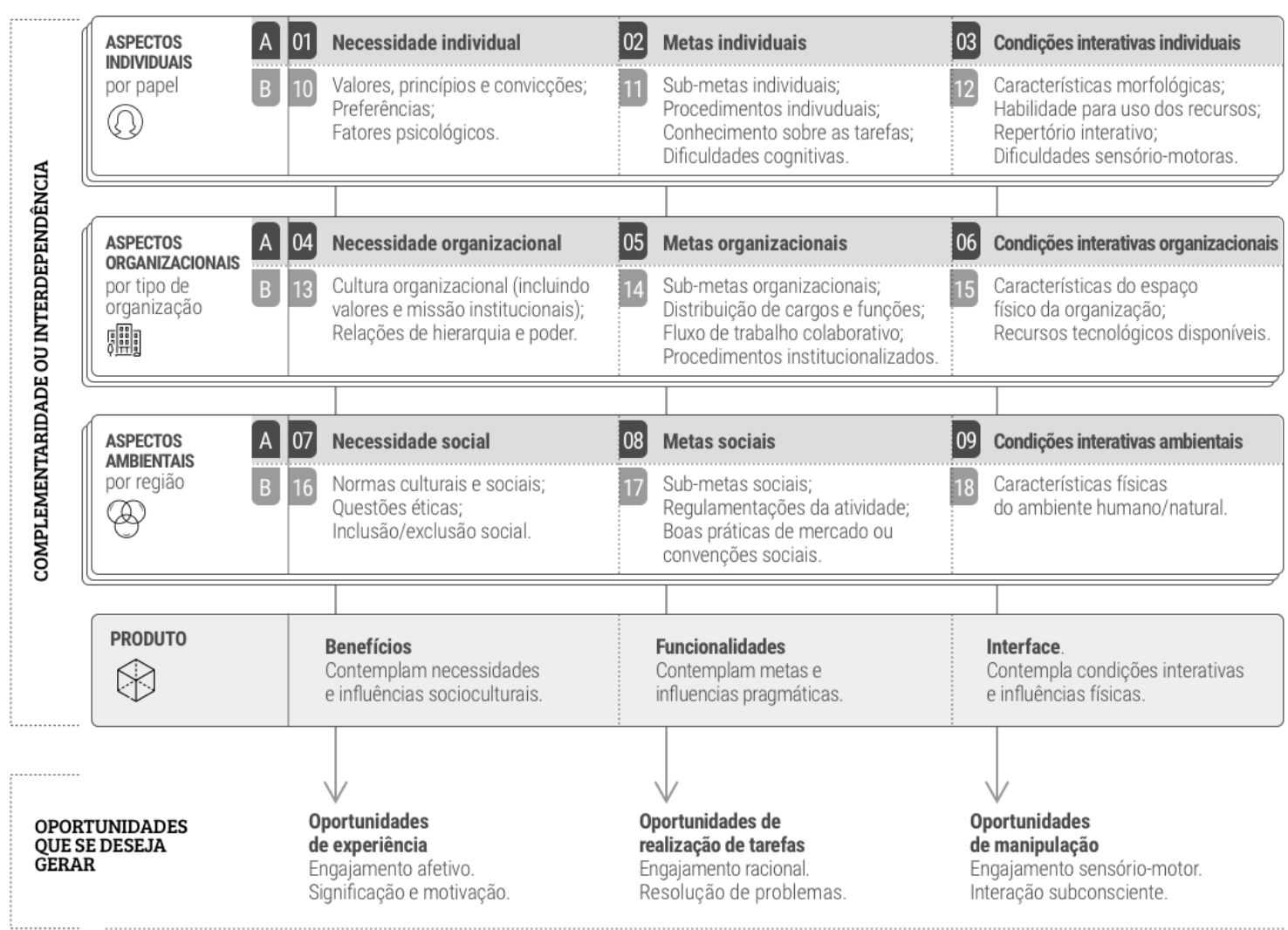

PROCESSO SUGERIDO PARA OUSO:

A VIS̃̃o GERAL DA ESTRUTURA DA ATIVIDADE

Identifique todos os envolvidos na atividade, incluindo indivíduos em diferentes papéis, bem como organizações e ambientes onde os indivíduos estão inseridos. A seguir, para cada papel, tipo de organização e ambiente identifique sua necessidade, sua meta geral e suas condições interativas.

\section{B MAPEAMENTO DAS CARACTERÍSTICAS DOS USUÁRIOS E DO CONTEXTO}

Organize as informações que você dispõe sobre cada tipo de usuário (papel), tipo de organização e ambiente onde ocorre a atividade. Devem ser informados os itens especificados na ferramenta. Você pode gerar hipóteses para os itens desconhecidos ou deixar em branco.

\section{ROTEIRO DE PESQUISA}

Elabore questões sobre as hipóteses a validar ou sobre os itens desconhecidos.

Figura 4 - Ferramenta de mapeamento proposta. Fonte: elaborada pelas autoras. 


\subsection{Aplicação e avaliação}

Participaram da aplicação quarenta e cinco alunos de graduação em design que cursavam entre a quarta e a sétima fases. A respeito das idades: (a) 17 participantes tinham menos de 21 anos; (b) 21 participantes tinham de 21 a 24 anos; (c) 7 participantes tinham mais que 25 anos. Sobre a experiência prévia: (a) 28 nunca tinham participado do projeto de aplicativos; (b) 17 já haviam projetado aplicativos, sejam em atividades acadêmicas ou no estágio/trabalho.

Para a realização da atividade, os alunos participantes se organizaram em duplas ou trios e cada equipe mapeou um sistema com pelo menos dois tipos de usuários inseridos em contextos organizacionais e sociais. Para facilitar a aplicação, a ferramenta foi entregue aos alunos no formato de canvas, no qual havia espaço para preenchimento de cada um dos itens mostrados na figura 4.

Após a aplicação da ferramenta, os alunos receberam um questionário com afirmações a respeito do uso da ferramenta e o responderam individualmente. Cada afirmação estava acompanhada de um item em uma escala likert de cinco níveis que incluíam as opções 'discordo fortemente', 'discordo', 'neutro', 'concordo' e 'concordo fortemente'. A respeito da visualização da estrutura geral da atividade, as afirmações eram: (E01) os conceitos de necessidade, meta e condições auxiliaram a refletir sobre o foco da atividade dos futuros usuários e a explorar a forma como cada papel será atendido pelo aplicativo; (E02) a ferramenta auxiliou a refletir sobre o relacionamento entre os futuros usuários, contribuindo para uma visão macro do projeto e do seu escopo; (E03) nesta etapa, a aplicação da ferramenta é fácil, simples e clara; (E04) o material de apoio desenvolvido facilita a compreensão e aplicação da ferramenta.

A respeito do mapeamento das características dos usuários e do contexto, as afirmações (M01), (M02) e (M03) diziam que a ferramenta auxiliou a refletir sobre as características, respectivamente, dos futuros usuários, do contexto organizacional e do ambiente, contribuindo para o seu mapeamento e para a identificação dos itens a serem pesquisados. A afirmação (M04) era: a ferramenta estimula uma visão abrangente da atividade. Por fim, (M05) e (M06) eram iguais à (E03) e (E04), abordando, respectivamente, facilidade de aplicação e material de apoio para essa etapa. A tabulação dos resultados é apresentada nas figuras 5 e 6.

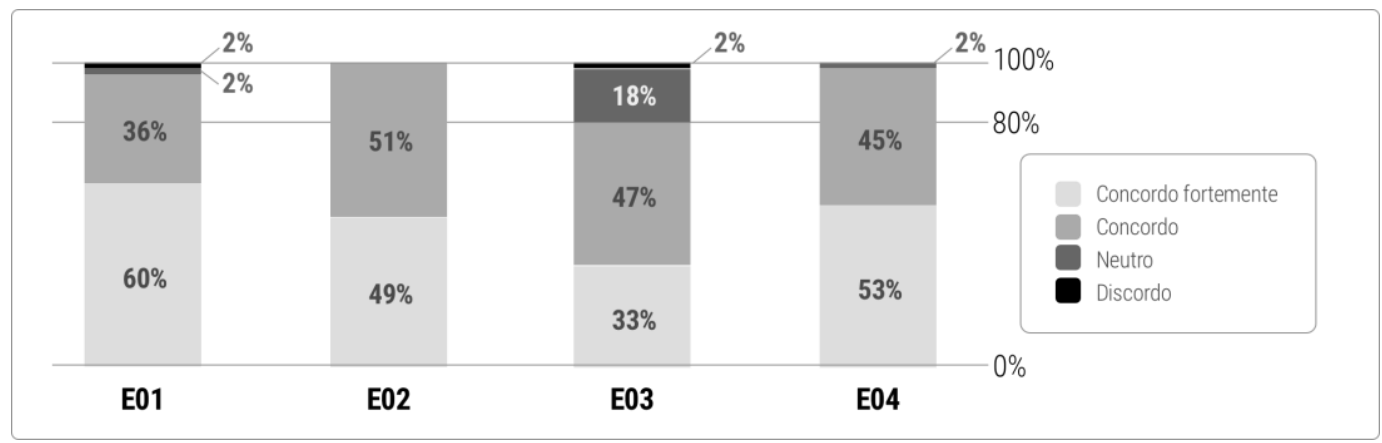

Figura 5 - Avaliação do mapeamento geral da estrutura da atividade. Fonte: elaborada pelas autoras. 


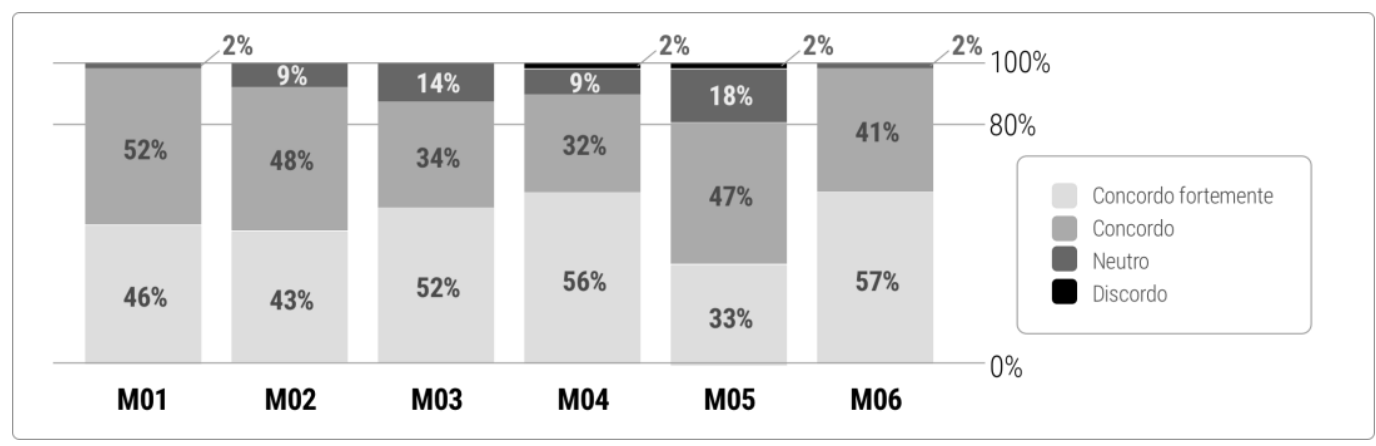

Figura 6 - Avaliação do mapeamento de características dos usuários e do contexto. Fonte: elaborada pelas autoras.

Os resultados numéricos demonstram uma percepção predominantemente positiva do uso da ferramenta. Em todos os itens, pelo menos $80 \%$ dos participantes escolheu as opções 'concordo' ou 'concordo fortemente'. Nenhum participante respondeu 'discordo fortemente'. Este resultado atende ao critério de Putnam et al. (1995), que define que a aprovação sobre um item de uma escala likert de cinco níveis é atingida quando oitenta por cento dos participantes votam nos dois níveis mais altos daquele item.

\section{Considerações finais}

O presente trabalho propôs uma ferramenta para o mapeamento da atividade para auxiliar designers a obterem uma visão mais aprofundada sobre os futuros usuários e a situação na qual o produto será utilizado. Esta ferramenta estimula uma visão sistêmica das dimensões afetiva, racional e sensório-motora da atividade. Além disso, a ferramenta vai além do nível individual e estimula a consideração das necessidades, das metas e das condições organizacionais e do ambiente sociocultural e físico.

Sobre os resultados obtidos com a avaliação, a expressiva concordância com a afirmação de que o uso dos conceitos de necessidade, meta e condições auxilia a identificar o foco da atividade dos futuros usuários e como devem ser atendidos pelo produto (item E01), revela o potencial da ferramenta em contribuir para a compreensão do domínio do problema de projeto. Por outro lado, mesmo os itens com menor índice de aprovação, referentes à facilidade de aplicação da ferramenta, demonstraram-se satisfatórios. Vale observar que os participantes da pesquisa estavam entre a quarta e a sétima fases do curso de graduação design. Assim, considerando o seu nível de experiência e a complexidade da tarefa de mapeamento, pode ser destacado o seu potencial didático. A ferramenta pode ser empregada em disciplinas de projeto em ambientes acadêmicos ou em cursos para profissionais que buscam compreender a pesquisa voltada à abordagem da experiência do usuário. Em ambos os casos, permite apresentar os itens que podem ser mapeados, descrevendo também o relacionamento entre eles. Além disso, seu uso prático na atividade de projeto guia a identificação das informações disponíveis no início do projeto, bem como das hipóteses que precisam ser validadas e dos itens desconhecidos que precisam ser pesquisados. Por fim, como sugestão de trabalhos futuros, pode-se citar a elaboração de outras ferramentas de projeto baseadas no conceito de atividade para contemplar as etapas de especificação funcional e avaliação de qualidade. 


\section{Referências}

BÆRENTSEN, K. B.; TRETTVIK, J. An activity theory approach to affordance. Proceedings of the second Nordic conference on Human-computer interaction.

Anais...Arhus, Denmark: ACM, 19 out. 2002.

BEDNY, G.; KARWOWSKI, W. Activity theory as a basis for the study of work. Ergonomics, v. 47, n. 2, p. 134-153, 2004.

CIAVOLA, B. T.; GERSHENSON, J. K. Affordance theory for engineering design. Research in Engineering Design, v. 27, n. 3, p. 251-263, 2016.

GALVAO, A. B.; SATO, K. Affordances in product architecture: Linking technical functions and users' tasks. ASME 2005 International, 2005.

GIBSON, J. J. The Ecological Approach to Visual Perception. Houghton Mifflin, 1979.

GRANGE, C.; BENBASAT, I. A guiding framework for developing theories investigating the design drivers of IT use and value. Proceedings of the JAIS Theory Development Workshop. Anais...2011

HARTSON, R. Cognitive, physical, sensory, and functional affordances in interaction design. Behaviour \& information technology, v. 22, n. 5, p. 315-338, 2003.

HASSENZAHL, M. The thing and I: understanding the relationship between user and product. In: BLYTHE, M. A. et al. (Eds.). . Funology: From Usability to Enjoyment. Human-Computer Interaction Series. Netherlands: Springer, 2004. v. 3p. 31-42.

HASSENZAHL, M. et al. Designing Moments of Meaning and Pleasure. Experience Design and Happiness. International Journal of Design, v. 7, n. 3, p. 21-31, 2013.

ISO. 9241-11: 2018 (en) ergonomics of human-system interaction - part 11: Usability: Definitions and concepts. International Organization for Standardization, Geneva, Switzerland, 2018.

JONIETZ, D.; TIMPF, S. On the relevance of Gibson's affordance concept for geographical information science (GISc). Cognitive processing, v. 16 Suppl 1, p. 265-269, set. 2015.

KAPTELININ, V.; NARDI, B. Affordances in HCI: toward a mediated action perspective. Proceedings of the SIGCHI Conference on Human Factors in Computing Systems. Anais...ACM, 2012.

KITCHENHAM, B.; CHARTERS, S. Guidelines for performing Syst Software Engineeri. [s.1.] Keele University and Durham University Joint Report, 2007.

LEONT'EV, A. N. Activity, Consciousness, and Personality. Englewood Cliffs: PrenticeHall, 1978.

NAHL, D. Social-biological information technology: An integrated conceptual framework. Journal of the American Society for Information Science. American Society for Information Science, v. 58, n. 13, p. 2021-2046, 2007.

POLS, A. J. K. Characterising affordances: The descriptions-of-affordances-model. Design Studies, v. 33, n. 2, p. 113-125, 2012.

PUCILLO, F.; CASCINI, G. A framework for user experience, needs and affordances. Design Studies, v. 35, n. 2, p. 160-179, 2014.

PUTNAM, J. W.; SPIEGEL, A. N.; BRUININKS, R. H. Future directions in education and inclusion of students with disabilities: A Delphi investigation. Exceptional children, v. 61, n. 6, p. 553-576, 1995.

ROZYCKI, E.; KELLER, S.; CYBULSKI, J. Business process affordances through the lens of activity theory. ACIS 2012: Location, location, location: Proceedings of the 23rd 
Australasian Conference on Information Systems 2012. Anais...ACIS, 2012.

SHARRITT, M. J. Evaluating Video Game Design and Interactivity. In: VAN ECK, R. (Ed.). Interdisciplinary Models and Tools for Serious Games. [s.1.] IGI Global, 2010. p. 177-205.

SUN, H. An Activity Approach to Cross-Cultural Design. In: AYKIN, N. (Ed.). . Usability and Internationalization. HCI and Culture. Lecture Notes in Computer Science. Berlin, Heidelberg: Springer Berlin Heidelberg, 2007. v. 4559p. 196-205.

SUN, H.; HART-DAVIDSON, W. F. Binding the Material and the Discursive with a Relational Approach of Affordances. Proceedings of the 32nd Annual ACM Conference on Human Factors in Computing Systems. Anais...: CHI '14.New York, NY, USA: ACM, 2014.

TURNER, P. Affordance as context. Interacting with computers, v. 17, n. 6, p. 787-800, 2005.

TURNER, P.; TURNER, S. An Affordance-based Framework for CVE Evaluation. In: XRISTINE FAULKNER B A PGCE; JANET FINLAY BA, M.; DÉTIENNE, F. (Eds.). . People and Computers XVI - Memorable Yet Invisible. [s.1.] Springer London, 2002. p. 89-103.

VICENTE, K. J.; RASMUSSEN, J. The Ecology of Human-Machine Systems II: Mediating "Direct Perception" in Complex Work Domains. Ecological psychology: a publication of the International Society for Ecological Psychology, v. 2, n. 3, p. 207-249, 1 set. 1990.

VYAS, D.; CHISALITA, C. M.; DIX, A. Organizational Affordances: A Structuration Theory Approach to Affordances. Interacting with computers, v. 29, n. 2, p. 117-131, 2017.

VYAS, D.; CHISALITA, C. M.; VAN DER VEER, G. C. Affordance in interaction. [s.l: s.n.]. v. 250

ZHANG, J.; PATEL, V. L. Distributed cognition, representation, and affordance. Cognition Distributed: How Cognitive Technology Extends Our Minds, v. 16, p. 137-144, 2008.

ZHANG, P. Motivational affordances: reasons for ICT design and use. Communications of the ACM, v. 51, n. 11, p. 145-147, 2008.

ZHAO, Y. et al. Conceptualizing perceived affordances in social media interaction design. Aslib proceedings, v. 65, n. 3, p. 289-303, 2013.

\section{Sobre as autoras}

\section{Genilda Oliveira de Araujo}

Doutoranda em Engenharia de Produção na linha de pesquisa de Ergonomia pela Universidade Federal de Santa Catarina (UFSC). Mestrado em Ciência da Computação pela Universidade Federal de Santa Catarina (UFSC). Graduação em Design Gráfico pela Universidade do Estado de Santa Catarina (UDESC) e Graduação em Ciência da Computação pela Universidade Federal do Maranhão (UFMA).

genilda@gmail.com

\section{Lizandra Garcia Lupi Vergara}

Professora Associada na Graduação (DEPS) e na Pós-Graduação (PPGEP) da Engenharia de Produção e na Pós-Graduação em Arquitetura (PosARQ) da Universidade Federal de Santa Catarina (UFSC). Doutorado e Mestrado em Ergonomia na Engenharia de Produção da Universidade Federal de Santa Catarina (UFSC). Graduação em Arquitetura e Urbanismo pela Universidade Estadual de Londrina (UEL).

1.vergara@ufsc.br 